\title{
UN CAMINO TRANSVERSAL AL QHAPAQ ÑAN EN EL INTERFLUVIO MOCHE-CHICAMA, COSTA NORTE DEL PERÚ
}

\author{
GORI TUMI ECHEVARRÍA LóPEZ \\ Aroueólogo. Universidad Nacional Mayor de SAN Marcos \\ goritumi@gmail.com \\ Víctor DaVid CoRcuera Cueva \\ Arqueólogo. UniVERSIDAd NACIONAL DE TRujILlo \\ victorcorcuera@gmail.com \\ EDUARDO LEÓn SALAZAR \\ ARQUITECTO. UNIVERSIDAD NACIONAL DETRUJILLO \\ eduardo.leon@hotmail.de
}

\section{RESUMEN}

El articulo describe y analiza un segmento del camino arqueológico que cruza el interfluvio entre los ríos Moche y Chicama, atravesando en su trayecto la confluencia de los cerros Piedra Parada y Portachuelo, siendo el único camino conocido en el área que usa la zona de montañas como parte de su vía siguiendo un trayecto general orientado Norte - Sur

A partir de las observaciones y hallazgos, los autores discuten las características constructivas y de diseño del camino, así como sus aspectos de correspondencia temporal y uso continuo. Se hace hincapié que esta es solo una sección del camino total, siendo una de las más relevantes de todo el sistema.

Palabras Clave: Capaq Ñan, camino, montañas, lomas, Moche.

\section{ABSTRACT}

The article describes and analyzes a segment of the archaeological road that crosses the area between the Moche and Chicama rivers, going through the confluence of the Piedra Parada and Portachuelo hills, being the only known in the zone that uses the area of mountains as part of their way following a North - South general orientation. 
From the observations and findings the authors discuss the construction and road design characteristics, as well as the aspects of temporal correspondence and continuous use. It is stresses that this is only a section of the road, being one of the most important of the entire system.

KeYwORDS: Capaq Ñam, road, mountains, lomas, Moche.

\section{INTRODUCCIÓN}

La zona interfluvial entre los valles de Moche y Chicama, en la costa norte del Perú, está dominada por una corta cadena de montañas que constituyen el más importante rasgo paisajístico del área, y cuyo carácter geográfico monumental ha constituido, por miles de años, un valor cultural de las ocupaciones humanas que se han sucedido hasta la actualidad en ese territorio (Franco 2012). Lamentablemente, en los últimos años, la expansión urbana e industrial en la provincia de Trujillo está afectando seriamente la integridad de estas montañas, que incluyen un muy complejo sistema ecológico y evidencias arqueológicas no antes exploradas. Entre las amenazas más contundentes se hallan la invasión de terrenos, la ubicación de galpones avícolas, la explotación minera y por sobre todo el trazado del canal madre del Proyecto CHAVIMOCHIC sobre la zona sur y oeste del Cerro Campana, la montaña más reconocida y prominente de la zona.

La investigación arqueológica independiente, realizada con el fin de documentar y proteger los diversos testimonios culturales existentes en estas montañas, ha logrado poner al descubierto una serie de evidencias, entre las que destacan sitios con arquitectura, cerámica, lítico, pictogramas, geoglifos, e incluso minas y altares ceremoniales (Echevarría y Corcuera 2011, Valladares 2012, Franco et. al. 2013); materiales que se identificaron sólo en los alrededores del cerro Campana.

La documentación de estos materiales no necesariamente ha agotado las posibilidades de hallar más testimonios arqueológicos, constituyendo básicamente un indicador importante de la enorme variación cultural y arqueológica que encierra el área y que todavía debe seguir siendo explorada. En ese sentido decidimos ampliar el área de observación, desde la cadena de montañas hacia el norte, evidenciando un camino arqueológico entre el Cerro Piedra Parada y el Cerro Portachuelo, e cual no había sido objeto de un estudio anterior detallado.

Este camino corta diagonalmente un ramal del Qhapac Ñan, que cruza el desierto costero entre los valles de Moche y Chicama, interconectando el litoral en la bahía de Huanchaco, la pampa desértica, y probablemente parte de la campiña del valle de Chicama, en dirección al valle medio. Esta configuración constituye un importante rasgo arqueológico de toda la zona, y en especial de las montañas que estamos estudiando.

\section{ANTECEDENTES}

El único reporte que se conoce acerca del camino que atraviesa los cerros del interfluvio Moche-Chicama ha sido elaborado por Coleen Beck (1979) para su tesis doctoral sobre los caminos antiguos de la costa norte del Perú. En este reporte se le asigna el número 14 de un total de 15 caminos, siendo el que atraviesa un paso montañoso hacia el oeste de cerro Campana, que es el área de sectorización para la identificación de caminos en la zona (Beck 1979). El paso que menciona Beck es en realidad un abra entre los cerros Piedra Parada y Portachuelo, que es atravesado por el camino en dirección general Norte-Sur. El camino sigue la sinuosidad de la topografía de la zona, claramente condicionada por las estribaciones rocosas bajas de las montañas mencionadas.

Beck nota adecuadamente que el camino, a partir del abra o paso, se pierde con dirección al norte, siendo mejor definido al lado opuesto, donde se encuentra "cortado profundamente, atravesando te- 
rreno difícil en su continuación al sur" (Beck 1979: 106-107; traducción nuestra). Asimismo al sur del abra, el autor ubica cuatro sitios arqueológicos, que identifica como "paravientos" (windscreen), los cuales registra con las letras "S", "T", "U" y "V" (Fig. 1); mencionando también presencia de cerámica Chimú asociada a la ruta de estudio. Según sus propios datos el camino tiene una orientación S2EN34O, siendo del tipo "parcialmente aclarado y en cuesta" (Beck 1979: 111), con un trayecto que atraviesa, desde el abra, la pampa al oeste de cerro Campana hasta la playa al norte de Huanchaco pueblo.

Beck estima que el camino es tardío, de filiación cultural Chimú-Inca para su ubicación más temprana, destacando que cruza otros caminos en el área (ver Fig. 1), específicamente los asignados con los números 10, 7, 4 y 9. Estos han sido relacionados todos a la época Chimú; y Chimú-Inca también para los caminos 7 y 10 (Beck 1979: 107). A partir de aquí no hay referencias más detalladas y la mayoría de las documentaciones sobre los caminos arqueológicos del área se han centrado en el camino principal, probadamente usado durante el Tawantinsuyu (Von Hagen 1975, Bernabé 2014, Hyslop 2014), y que en Beck (1979) aparece con los números 12 y 10 para la zona oeste del cerro Campana. El Qhapac Ñam destaca por su fragmentación, variación técnica y variación formal (Bernabé 2014, Hyslop 2014), constituyendo un camino completamente diferente al ejemplo que estamos refiriendo.

El camino por el abra de los cerros Piedra Parada y Portachuelo es usado en la actualidad, como se atestigua por las numerosas huellas dejadas por los transeúntes y vehículos todo terreno en varias secciones de su trayecto, y constituye una ruta pedestre reconocida incluso en la carta nacional, como se puede verificar en la carta de Chocope, hoja 1055 (16-e). Desde la zona de playa este camino sigue un trayecto ininterrumpido hasta el abra, reconociéndose solamente hacia el lado sur y oeste de la cadena de montañas entre los valles de Moche y Chicama.

\section{EL ENTORNO GEOGRÁFICO}

El camino se encuentra dentro de la región Chala (Pulgar 1946), en el interfluvio desértico de los ríos Chicama y Moche, con una geomorfología contrastada cuyo rasgo más destacado es la existencia de una corta cadena de cerros de baja altitud que forman los cerros Piedra Parada, Portachuelo, La cumbre y Campana (Fig. 2). La cadena de cerros tiene una orientación general noroeste-sureste, y una altura mayor de aproximadamente $1000 \mathrm{~m}$ en el cerro Campana y aproximadamente $500 \mathrm{~m}$ para el cerro Piedra Parada. Todos estos cerros muestran superficialmente evidencias de procesos de denudación y degradación, como desprendimientos, acumulaciones aluviónicas, y a menor escala procesos de meteorización y erosión sobre las rocas.

Hacia el sur y oeste de esta cadena montañosa el viento ha transportado arena y ha cubierto de una manera casi homogénea la zona circundante, que es una pampa desértica. La arena ha generado un paisaje ondulado en ascenso hacia las faldas bajas de los cerros, en especial del cerro Campana, donde sirve de sustrato a las lomas estacionarias de la región. La cobertura de arenas, desde el litoral, se interrumpe cuando es cortada por las pequeñas quebradas que descienden de los cerros, que van desde escorrentías hasta verdaderos huaycos, y pequeñas y cortas cuencas secas. Hacia la zona del abra, entre los cerros Piedra Parada y Portachuelo, estas características son bastante marcadas ya que la zona está dentro de una hoyada formada por los contornos de los tres principales cerros, dejando el abra como el elemento más bajo de la confluencia, separando el cerro Piedra Parada de las demás montañas.

Hacia la zona oeste, desde el abra misma, el camino utiliza tanto el sustrato rocoso del cerro como las dunas formadas por la acumulación eólica para soportar su trazo. Hacia el norte el abra cae en una quebrada que baja súbitamente hacia la pampa, estando enmarcada entre las estribaciones rocosas 
de la montaña. Esta misma quebrada fue usada como camino durante tiempos arqueológicos, terminando su trayecto hacia la pampa, que, debido a que esta fuera de la influencia directa del viento y su carga de arena, se presenta en un nivel más bajo y sin el talud constante de la parte oeste de las montañas.

El camino es cortado en su proyección sur por una quebrada de casi un kilómetro de ancho que baja de la ladera oeste del Cerro Campana. Esta quebrada muestra en su superficie arena gruesa que ha sido transportada por el agua y también depositada por el viento. Después de la quebrada, hacia el sur, el camino corta la zona de dunas entrando al área de lomas con vegetación xerófita, achupallas, atravesando el tablazo de la Pampa el Alto, hasta prácticamente el litoral inmediatamente al norte de Huanchaco.

\section{El Camino}

Como ya hemos advertido, la zona en la que se hizo el reconocimiento cubre la parte norte del abra entre los cerros Piedra Parada y Portachuelo, la zona superior del abra; y hacia el sur, todo el trayecto del camino hasta el borde sur de la quebrada seca que corta la pampa, y que se origina en la base oeste y sur del cerro campana (Fig. 3). Esta sección cubre una longitud aproximada de $3 \mathrm{~km}$ lineales, y es solo un fragmento del camino, desde el ingreso por el lado norte del abra, que en total tiene al menos $14 \mathrm{~km}$ de longitud con un eje de orientación general N-S

La sección de camino revisada presenta, como ya dijimos, una orientación general N-S, variando algunos grados de acuerdo a la topografía del terreno sobre la que se adapta. El camino es claramente reconocido a partir de su entrada por el lado norte del abra, y se delimita asimismo mediante los contornos de la quebrada rocosa que lo forma. A partir del abra hacia el sur, el camino se acerca hacia el talud bajo de la ladera este del cerro Piedra Parada para salir de la influencia de la montaña y su talud arenoso, adentrándose hacia la quebrada seca y luego penetrar la extensa pampa de arena del interfluvio Moche -Chicama. De acuerdo a esto se puede subdividir la sección en tres segmentos separados, con características morfológicas y técnicas bastante diferenciadas:

- Segmento del abra

- Segmento en la ladera del Cerro Piedra Parada

- Segmento en la quebrada seca

- Segmento del abra

Este segmento comprende la sección norte del abra, desde la entrada a la quebrada que conduce a la cima de la confluencia, y la zona sur hasta la definición del camino sobre la ladera del Cerro Piedra Parada. Hacia la parte norte el camino es un sendero naturalmente delimitado cuya superficie está conformada por arena eólica, la misma que cambia a roca expuesta por la erosión y la intemperie mientras se asciende hacia la cima del abra. Esta parte no contiene mucha arena debido a que el viento reinante no impacta directamente sobre esta sección de la montaña. Desde el norte el camino se presenta como una abertura encajonada (Fig. 4), pero se abre inmediatamente mientras se sube al abra (Fig. 5), donde el panorama al sur se muestra como el de una hoyada extensa, por lo que el camino se pliega hacia la ladera baja del Cerro Piedra Parada.

En dirección al sur e inmediatamente al abra, a una altura de $252 \mathrm{msnm}$, el camino desciende sobre un relieve irregular formado por bancos de arena y salientes rocosas. El camino avanza de manera sinuosa pegándose a la ladera del Cerro Piedra Parada (Fig. 6) hasta proyectarse independientemente 
sobre la ladera del mismo cerro. El camino no tiene una clara definición hasta la saliente de un afloramiento de roca (Figs. 7 y 8) presentando un ancho variable. Hasta aquí el camino parece haberse asentado mediante el uso continuo más que mediante una delimitación técnica, pero también es evidente que su deterioro y falta de mantenimiento no ha ayudado a conservar su definición técnica.

El camino cruza la última saliente de roca del segmento ya sea por la parte alta, ascendiendo directamente o rodeándola sobre el mismo nivel horizontal. Debido a la irregularidad del trazo que la rodea creemos que el camino original ascendía la roca y bajaba mediante un terraplén construido, rasgo que marca el inicio del segundo segmento del camino.

\section{Segmento ubicado en la ladera del Cerro Piedra Parada}

El afloramiento de roca es el rasgo geológico que divide el primer y segundo segmento del camino. Ya sea cruzando sobre el afloramiento o rodeándolo, los trazos generados se unen pasando el elemento rocoso y es a partir de aquí donde el camino empieza a tener una clara definición técnica, ubicándose en la falda baja del cerro Piedra Parada y usando terraplenes como base estructural para su tendido (Fig. 9). El ancho promedio del camino luego de la roca es de 6 metros y por partes presenta expuestas rocas sueltas distribuidas sobre la calzada (Fig. 10), que consideramos constituyen elementos de la subestructura del terraplén. La formalidad en el ancho y la trayectoria es bastante destacable en esta parte, incluyendo el trazo sinuoso y semicircular de algunas partes de este trayecto (Fig. 11).

El camino desciende suavemente hasta un pequeño promontorio de arena y roca, que es cruzado mediante una sección aparentemente excavada junto a la ladera baja del cerro (Fig. 12), donde se hallaron varias edificaciones arqueológicas. Luego de este paso el camino sigue descendiendo manteniendo su configuración hasta llegar a otro promontorio bajo, también asociado a edificaciones, desde donde vuelve a descender abriéndose gradualmente hasta perder definición técnica; a partir de aquí el camino solo usa el sustrato geológico del suelo hasta la quebrada. Todo el trayecto descrito presentó una superficie de arena eólica y no una calzada preparada propiamente dicha. La parte estructural más destacada es la construcción del terraplén pegado a la ladera del cerro y las partes con piedras distribuidas en la superficie del camino, muchas de las cuales están disturbadas. No hay evidencia de construcciones laterales, muros, o marcas de camino específicas.

\section{Segmento en la quebrada seca}

Este segmento se origina en el corte que hace el camino sobre un pequeño promontorio natural hacia el este de las laderas bajas del cerro Piedra Parada. Como ya dijimos, a partir de aquí el camino se abre completamente perdiendo definición (Fig. 13) introduciéndose directamente sobre el cauce del río seco, donde se pierde visualmente. No obstante esto, el camino se proyecta naturalmente en forma lineal hasta la abertura del borde izquierdo -sur- de la quebrada seca (Fig. 14), siguiendo un rumbo Norte-Sur definido; de esta forma el camino retoma su trayecto cuando se introduce en los bancos de arena y la zona de lomas al oeste del Cerro Campana.

\section{Otras evidencias}

Se hallaron varios sitios arqueológicos independientes a lo largo de esta sección del camino. El más relevante de ellos se trata de un cementerio arqueológico guaqueado de la época Moche (Fig. 15), donde todavía han quedado remanentes numerosos fragmentos de cerámica (Fig. 16) y otras evidencias muebles. Este cementerio se halla en una hondonada entre dos colinas o cerros bajos, hacia la izquierda de la pequeña quebrada que baja del abra, casi a la altura de donde empieza el segundo segmento del camino descrito. 
Por otro lado también se reconocieron varias edificaciones arqueológicas sobre y cerca los dos promontorios naturales que se hallan consecutivamente al final del trayecto del segundo segmento de camino descrito (Figs. 17 y 18). Las edificaciones son recintos con muros bajos de roca en planta cuadrangular, en forma de L, e incluso muros a modo de pequeñas terrazas. En varias secciones del camino se halló cerámica dispersa en baja densidad, algunas piezas al parecer arrojadas por guaqueros (Fig. 19).

\section{ANÁLISIS Y DISCUSIÓN}

La documentación de este camino ayuda a establecer con más contundencia la enorme variedad de evidencia arqueológica presente en la cadena de montañas más allá del Cerro Campana, donde podemos incluir otros tipos de materiales y contextos arqueológicos, tal como cementerios y edificaciones antiguas. El registro de estos materiales testimonia la necesidad de emprender nuevos y más extendidos estudios en la zona de influencia de la cadena de montañas, las que pueden ser irreversible afectadas por las obras de infraestructura que se planean en el área, especialmente por el trazado del canal del Proyecto CHAVIMOCHIC, lotizaciones particulares y usos industriales diversos.

Observando la vía propiamente, podemos concluir que se trata de un camino de primer orden cuyo trazo ha sido adecuadamente planteado, utilizando técnicas de construcción complejas como el terraplenado de las laderas y la colocación de piedras como base subestructural de las calzadas, aprovechando además las condiciones naturales de la topografía del área; especialmente hacia toda el abra y sus contornos, y hacia la saliente de la quebrada seca, donde el camino se proyecta mediante un trayecto visual lineal uniforme.

El segundo segmento examinado es especialmente destacable ya que muestra un nivel técnico de elaboración sobresaliente, tanto en el aspecto constructivo, como en la parte del trazo elaborado, que sigue una línea semicircular bien definida (ver Foto 11). Para la elaboración del terraplén se usó arena eólica y las bases del talud preexistentes a lo que se sumó, al menos en algunas partes, según se observa, capas de piedras de campo que parecen haber servido como elementos superiores de la estructura del terraplén antes del tendido de la calzada, que pudo haber sido de arena como en la actualidad.

Como se ha advertido, previo y posterior al segundo segmento, el camino no muestra una pura uniformidad técnica y de diseño, lo que parece deberse a la gran variación topográfica y geomorfológica de esta sección específica, lo cual es interesante de considerar respecto de la manera como se trazó y planificó constructivamente el camino. Es posible considerar que los que proyectaron la vía han seleccionado solo algunas partes del mismo para un tratamiento especial, sin considerar el diseño completo con el mismo ardid técnico, es decir que secciones con segmentos estables y definidos, como el abra y la bajada este del cerro, no requirieron mayor tratamiento debido a la estabilidad, uniformidad y condicionamiento natural de la ruta en esa parte, que contrasta con la sección ladeada, inestable e irregular, de un trazo sobre la ladera de una pendiente arenosa, la que obligo a edificar el camino en este terreno. Por su parte el trazo sobre la quebrada, aparentemente no requirió un tratamiento técnico mayor, dada la inestabilidad de la zona y la posibilidad simple de una direccionalidad visual en un terreno abierto.

La variación técnica descrita en este caso parece estar condicionada por la geomorfología del terreno donde se trazó el camino, más que a una diferenciación temporal o cultural, como parece ser la que se manifiesta por ejemplo en el trazo del camino longitudinal entre el valle del Moche y Chicama, caminos 12 y 10 de Beck (1979), que se caracteriza por una gran variación técnica (Bernabé 2014, Hyslop 2014). Dada la uniformidad geomorfológica de la zona por la que atraviesa este camino 
principal, es difícil sostener que su manufactura y cronología sean uniformes, debiéndose buscar una explicación particular a estas diferencias.

En nuestro caso, si la explicación de la variación técnica y de diseño del camino es correcta, todo el trayecto de la sección estudiada puede estimarse contemporánea entre si desde su origen o edificación, sin considerar posibles remodelaciones o usos tardíos. En este sentido es muy posible que la antigüedad mayor de este monumento pueda retrotraerse a la época Moche, dada la existencia de un cementerio asociado a esta cultura a pocos metros del camino, dentro de los recodos de las colinas y montañas bajas de la hoya entre los Cerros Piedra Parada y Portachuelo. La ubicación tan localizada del cementerio y la del camino en el abra hace imposible no considerar que esta sea una ruta especifica del tráfico de personas y bienes entre los valles de Chicama y Moche durante el Intermedio Temprano del valle; lo que contrasta con la estimación de Beck (1979) de que el camino es de origen Chimú.

Beck había advertido restos de cerámica tardía a lo largo del camino, además de pequeñas edificaciones, pero su criterio de asignación temporal ha dependido meramente a la existencia de materiales aleatorios o muebles en el camino. La aproximación de Beck es todavía bastante estándar para este tipo de monumentos arqueológicos, los que aún se correlacionan mediante asociaciones espaciales, lo que no es un criterio científico en sentido estricto. Una asociación no constituye una prueba de nada, sino un mero hecho factico y no debería establecer una referencia temporal en ninguna dirección material específica. Si los materiales muebles no ayudan, como estamos tratando de establecer, la referencia temporal debe apuntar a la ocupación más antigua conocida para la zona, que puede incluso llegar a la Época Chavín o Cupisnique del interfluvio, pero que en el abra parece remitirse directamente al Intermedio Temprano. Si este uso ceremonial del área, por el cementerio, implicó un continuo tránsito de la zona por una salida natural definida, es entonces posible que la referencia temporal mínima para el origen del camino se establezca hacia el Intermedio Temprano, por lo que las evidencias muebles deben indicar un uso continuo hasta la época del Tahuantinsuyu.

Un camino no puede referenciarse como una particular pertenencia cultural. Si el origen fue Moche, como proponemos, culturalmente el camino debió ser usado por todas las sociedades posteriores tal como atestiguan sus materiales culturales, en este caso la evidencia más elocuente es la Chimú por la cerámica dispersa en la zona; pero esto no ha limitado su uso y el camino sigue vigente hasta hoy como hemos podido verificar por las huellas de uso actuales y su inclusión en las referencias de trochas de la carta nacional vigente. En la actualidad el corte de la proyección natural del camino hacia el norte, en dirección al pueblo de Chicama en el valle del mismo nombre, debido a la parcelación de los terrenos al norte y este del cerro El Portachuelo y Piedra Parada debe implicar probablemente el cierre definitivo de esta milenaria ruta, y quizá seamos los últimos testigos de su uso.

\section{CONCLUSIONES}

Este es todavía un reporte fragmentario, que trata únicamente con una parte del camino N-S que cruza el interfluvio de los valles Moche y Chicama a través de la cadena de montañas formadas por los cerros Campana, La Cumbre, Portachuelo y Piedra Parada. Preferimos cubrir preliminarmente una parte de este camino ya que el mismo puede ser dividido en dos grandes secciones según la zona por donde esta trazado: ya sea la zona de lomas y el arenal desértico de la pampa El Alto; o la zona de influencia de los cerros Portachuelo y Piedra Parada, y el abra formada por la unión de los mismos. Como vimos, esta última sección es bastante interesante en términos de una definición técnica de la vía, permitiendo además establecer una discusión preliminar sobre el origen y uso de este camino a partir de la interesante y variada información que hemos podido recuperar de su observación directa. 
Los caminos arqueológicos son evidencias muy complejas, tanto por su connotación constructiva y de diseño, así como por la dificultad intrínseca de poder ser fechados y de entender su permanencia, transformación y uso durante la historia de los pueblos a partir de su edificación originaria. Tal como demuestra Beck en su registro de caminos de la parte oeste del Cerro Campana, la complejidad constructiva y de diseño, y el trazo de numerosas y nuevas rutas de comunicación en esta pequeña sección del interfluvio Moche -Chicama, demuestran que estamos ante un panorama de enorme dinamismo social, y la ruta del abra es parte de ese gran y extendido sistema social que ha perdurado en el tiempo hasta hoy.

\section{BiBLIOGRAFÍA}

BECK, Coleen M.

1979 Ancient Road son the North Coast of Peru. University of California, Berkeley.

BERNABÉ ROMERO, Joseph A.

2014 El camino de los llanos: entre los valles de Chicama y Moche. Disponible en línea en: http://qhapaqnan.cultura.pe/sites/default/files/articulos/Articulo-Chicama-Moche.pdf [Consultado 20/5/15].

ECHEVARRÍA LÓPEZ, Gori Tumi y Víctor CORCUERA CUEVA

2011 Los geoglifos de Cerro Campana, segundo informe. Boletín APAR 3(10): 294-308. Lima.

FRANCO JORDÁN, Régulo

2012 El Apu Campana, la montaña sagrada Moche. Pueblo y Continente 23(5): 292-307. TrujilloFranco Jordán, Régulo; Carlos Quiroz Moreno, Percy Valladares Huamanchumo y Carlos Quiroz Gutierrez

2013 El Apu Campana, La montaña de las escenas de sacrificios humanos, historia, arqueología y biodiversidad. SIAN Revista Arqueológica 18(24). Trujillo.

HYSLOP, John

2014 Qhapac Ñam. El Sistema Vial Inkaiko. Ediciones Copé, Lima.

PULGAR VIDAL, Javier

1946 Historia y Geografía del Perú, Tomo I, Las Ocho Regiones Naturales del Perú. Universidad Nacional Mayor de San Marcos, Lima.

VALLADARES HUAMANCHUMO, Percy

2012 Quilca en el distrito de Huanchaco, La Libertad. Boletín APAR 3(11): 395-396. Lima

VON HAGEN, Victor W.

1975 Highway of the Sun. A Search for the Royal Roads of the Incas. Plata Publishing LTD, Great Britain. 


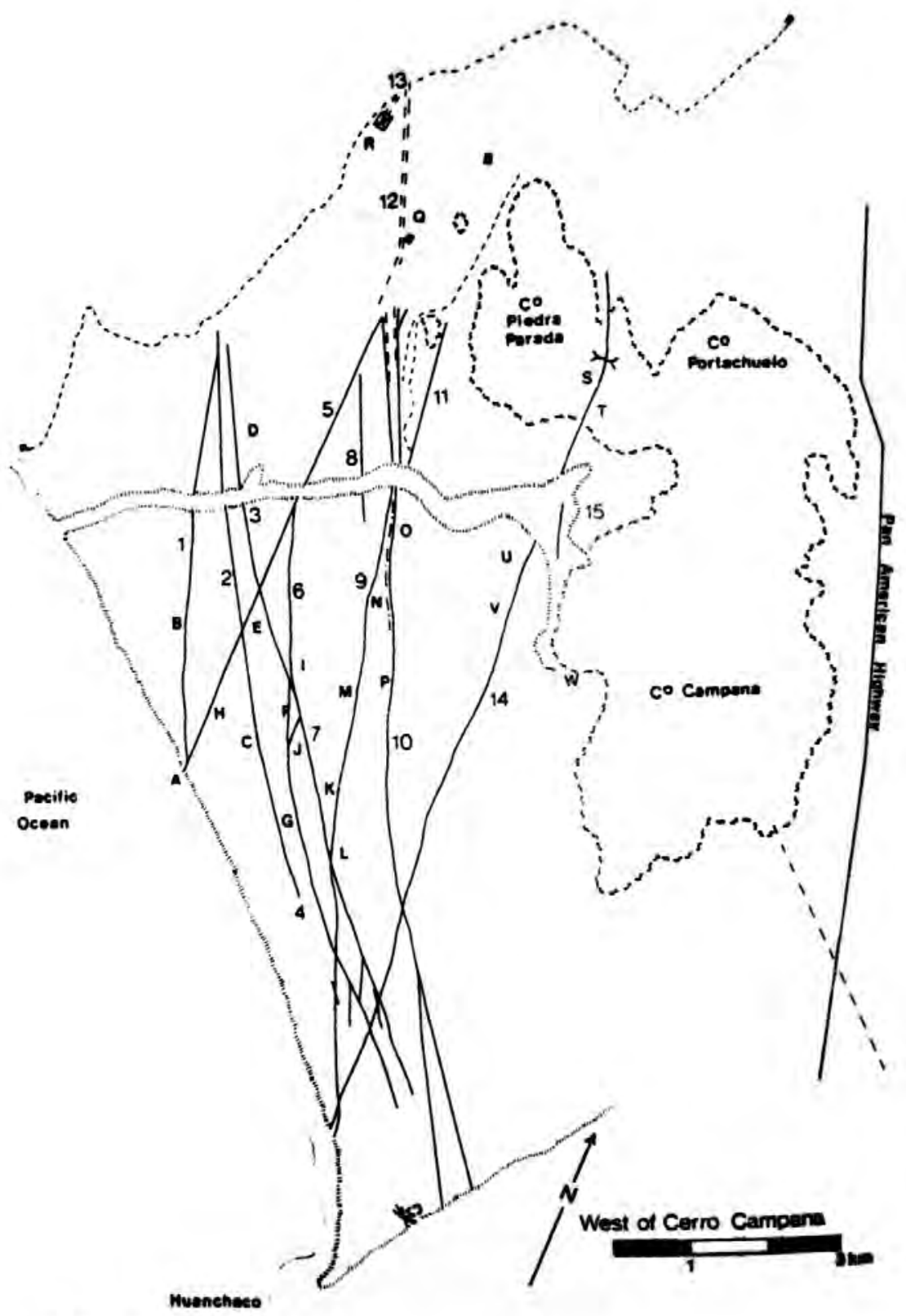

Pigure 17

Figura 1: Mapa de caminos de la zona oeste de Cerro Campana, interfluvio Moche-Chicama. Tomado de Beck (1979) 


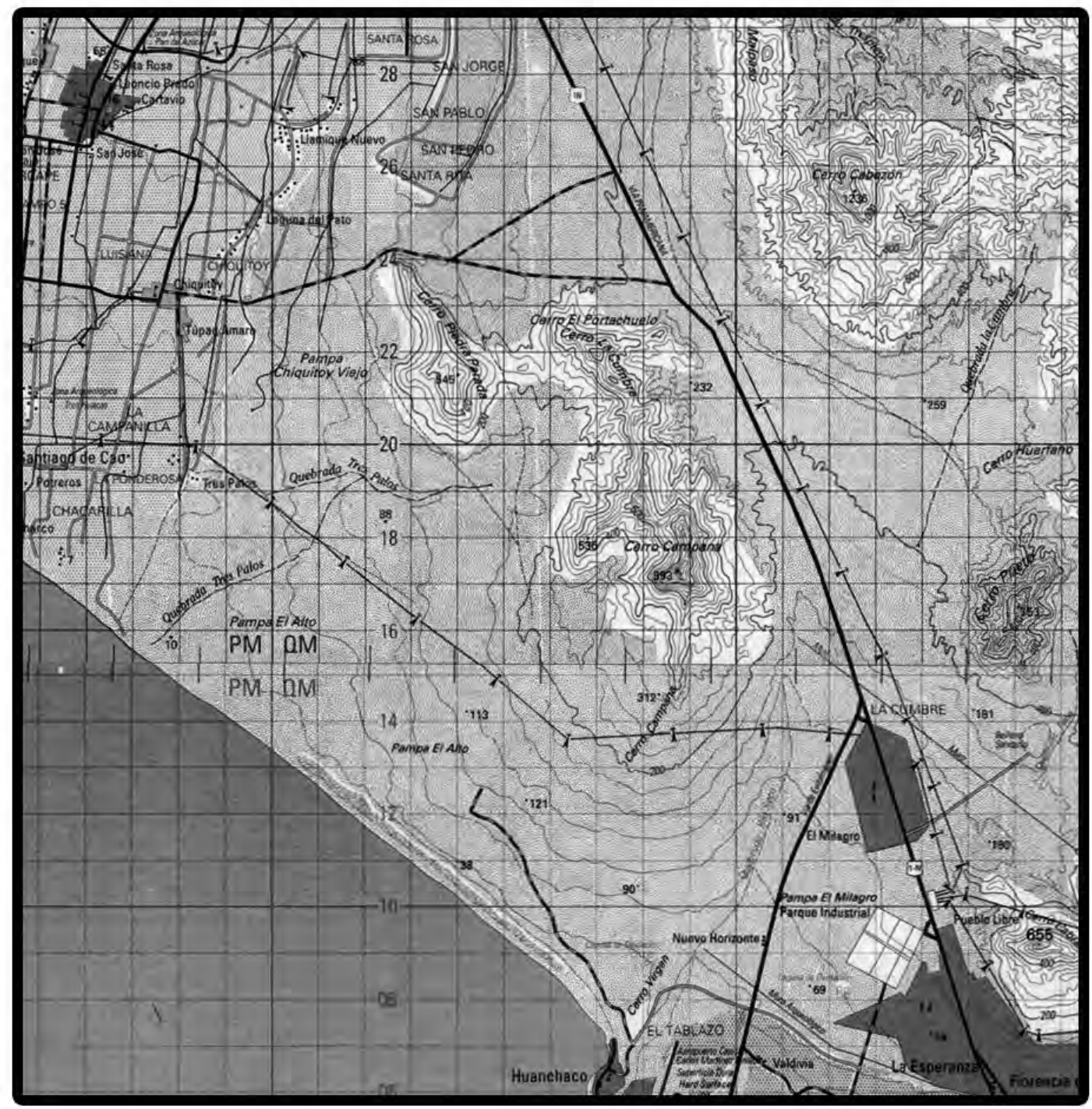

Figura 2: Mapa del interfluvio Moche - Chicama. Basado en la Carta Nacional hojas 16e-17e, escala 1:100000. IGN. 


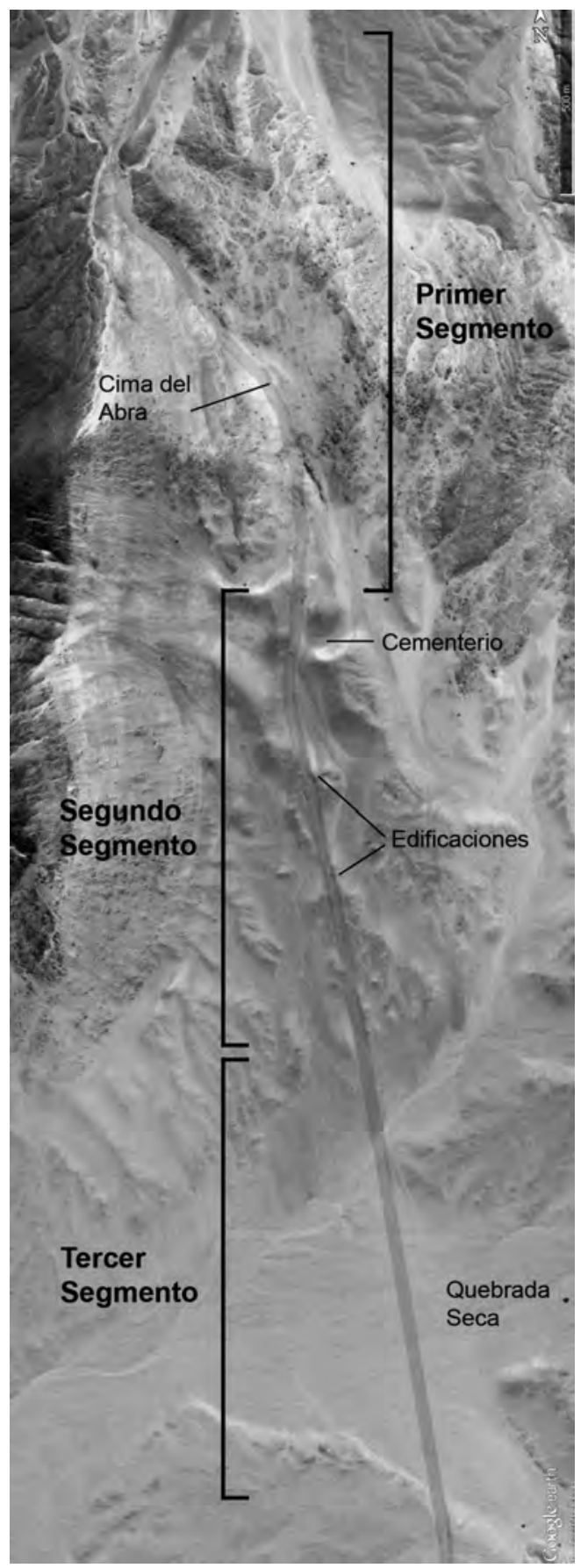

Figura 3: Área de reconocimiento, y sitios arqueológicos mencionados en este artículo. Tomado de Google Earth 2015. 


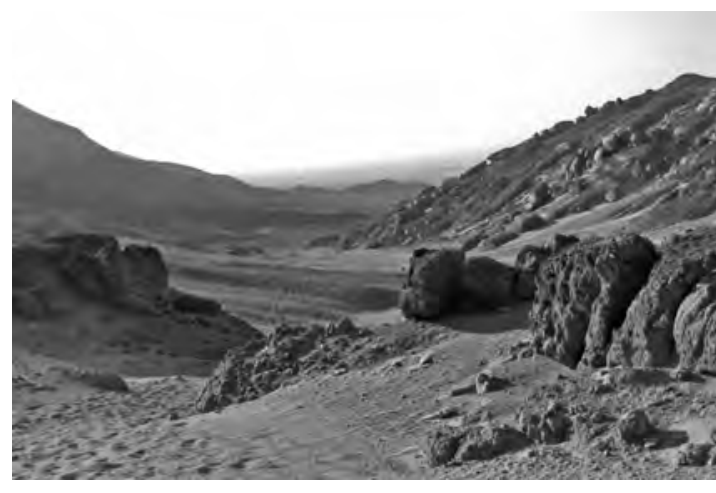

Figura 4: Parte baja del abra en dirección al norte, zona con ruta delimitada naturalmente.

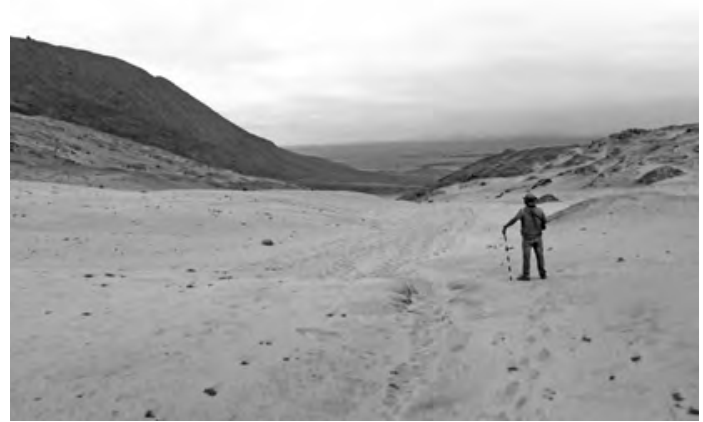

Figura 5: Vista del camino sobre el abra.

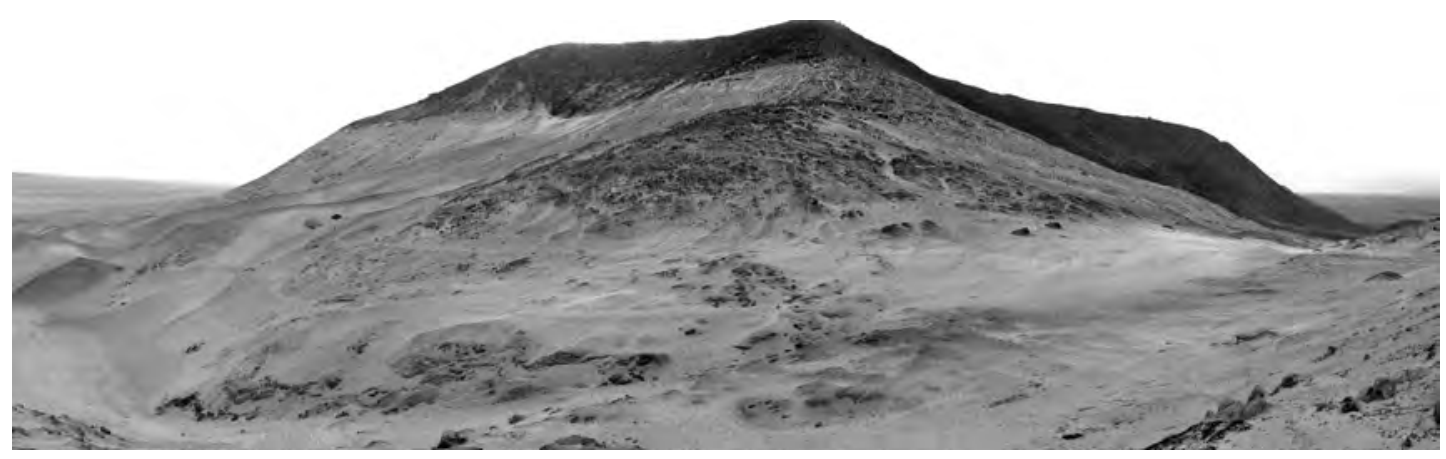

Figura 6: Segmento del camino sobre la ladera del cerro Piedra Parada.

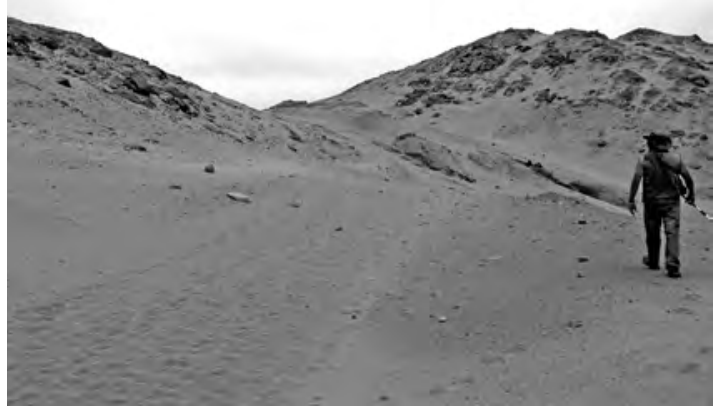

Figura 7: Vista del camino que baja del abra antes del espolón de roca.

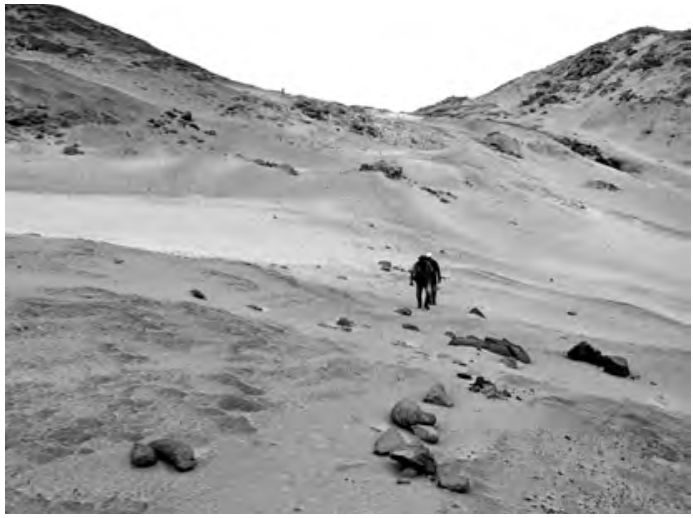

Figura 8: Vista del camino que baja del abra antes del espolón de roca. 


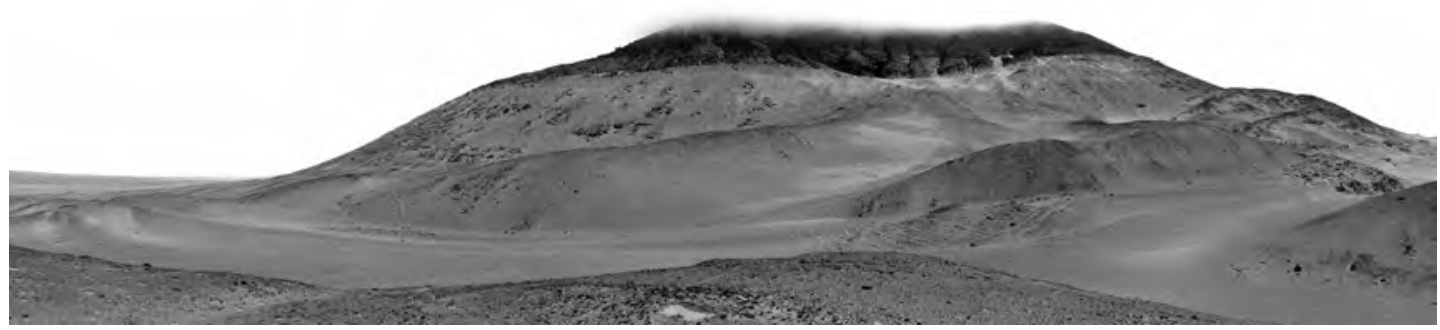

Figura 9: Panorama del segundo segmento del camino.

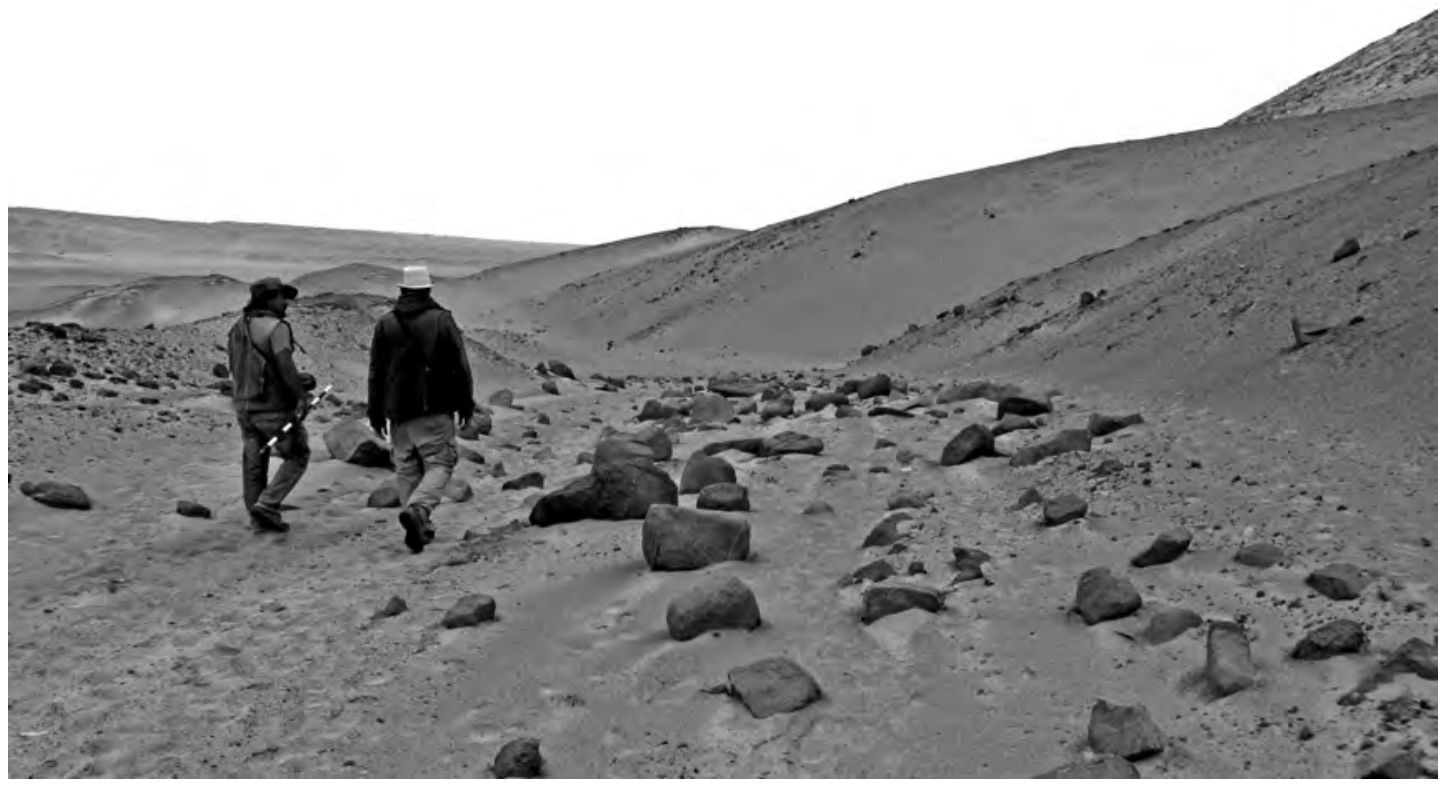

Figura 10: Rocas sueltas sobre el camino.

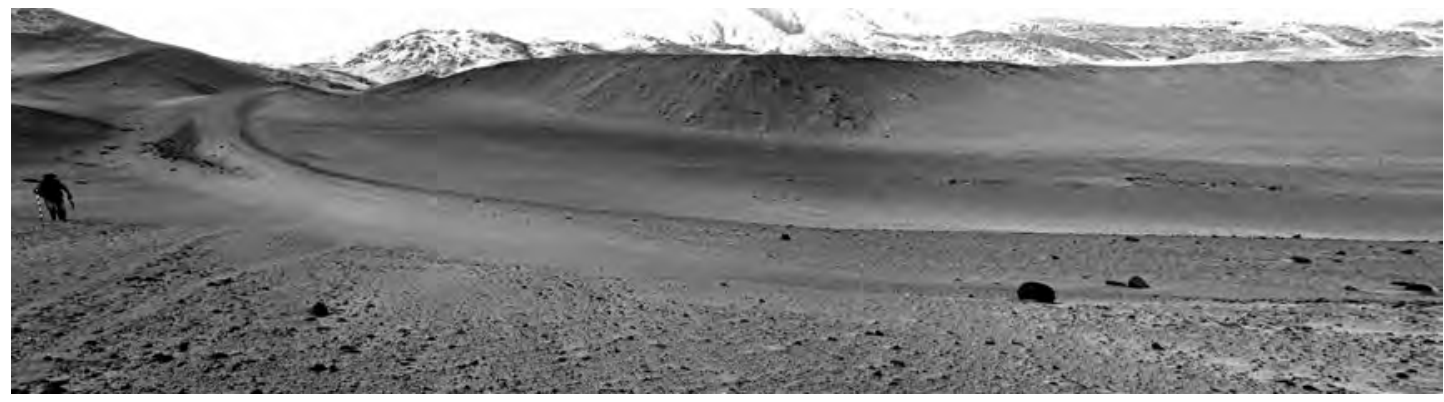

Figura 11: Trazo semicircular del camino terraplenado. 


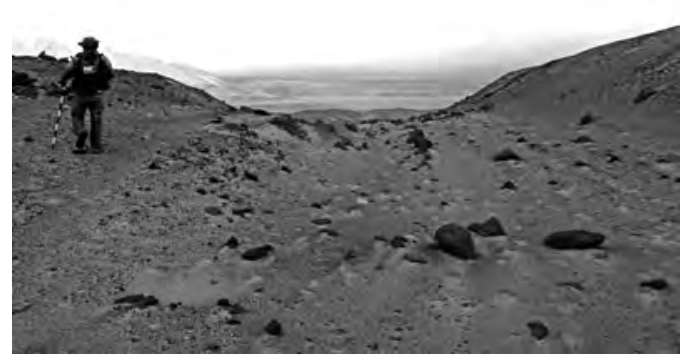

Figura 12. Sección excava del camino hacia el sur del segundo segmento.

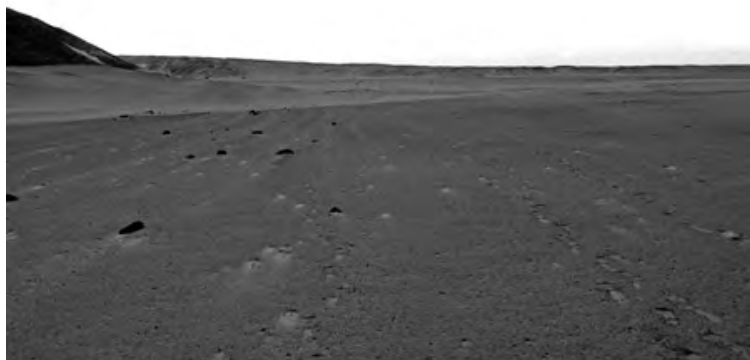

Figura 13. Panorama del tercer segmento del camino con vista al sur, que no presenta delimitación.

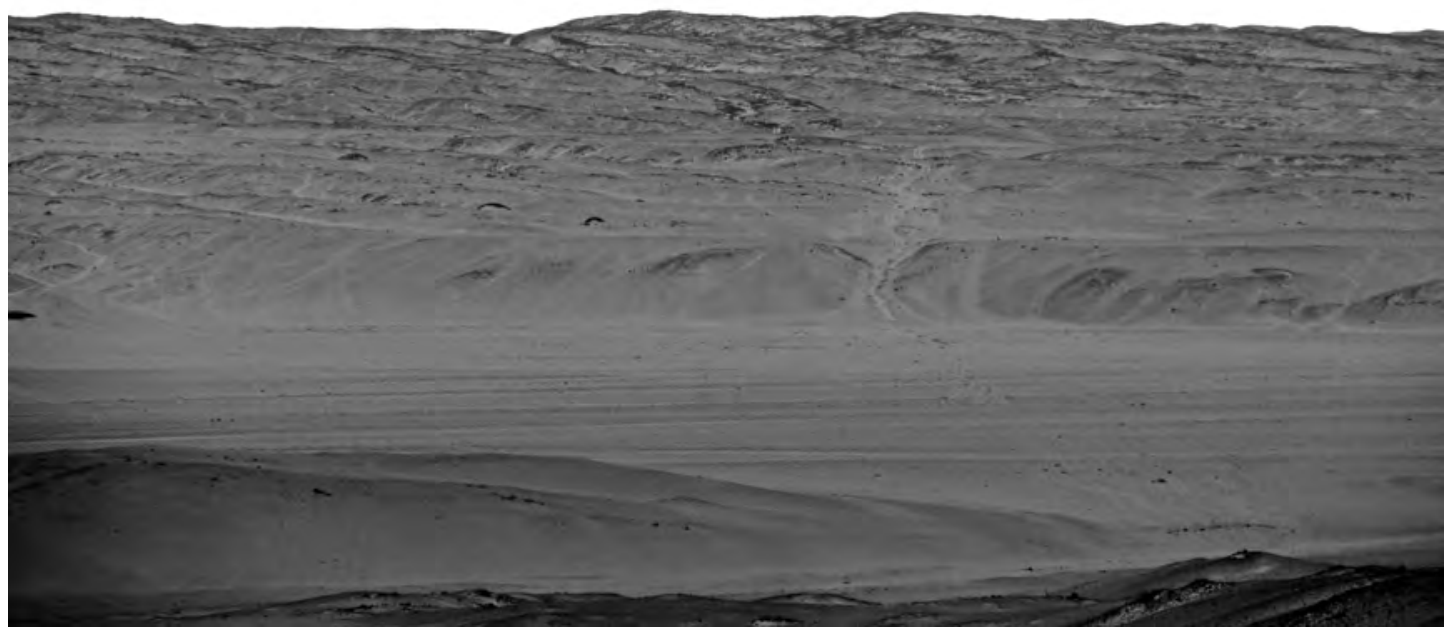

Figura 14. Corte del camino hacia el sur, entrando en la zona de dunas y lomas, finalizando el tercer segmento.

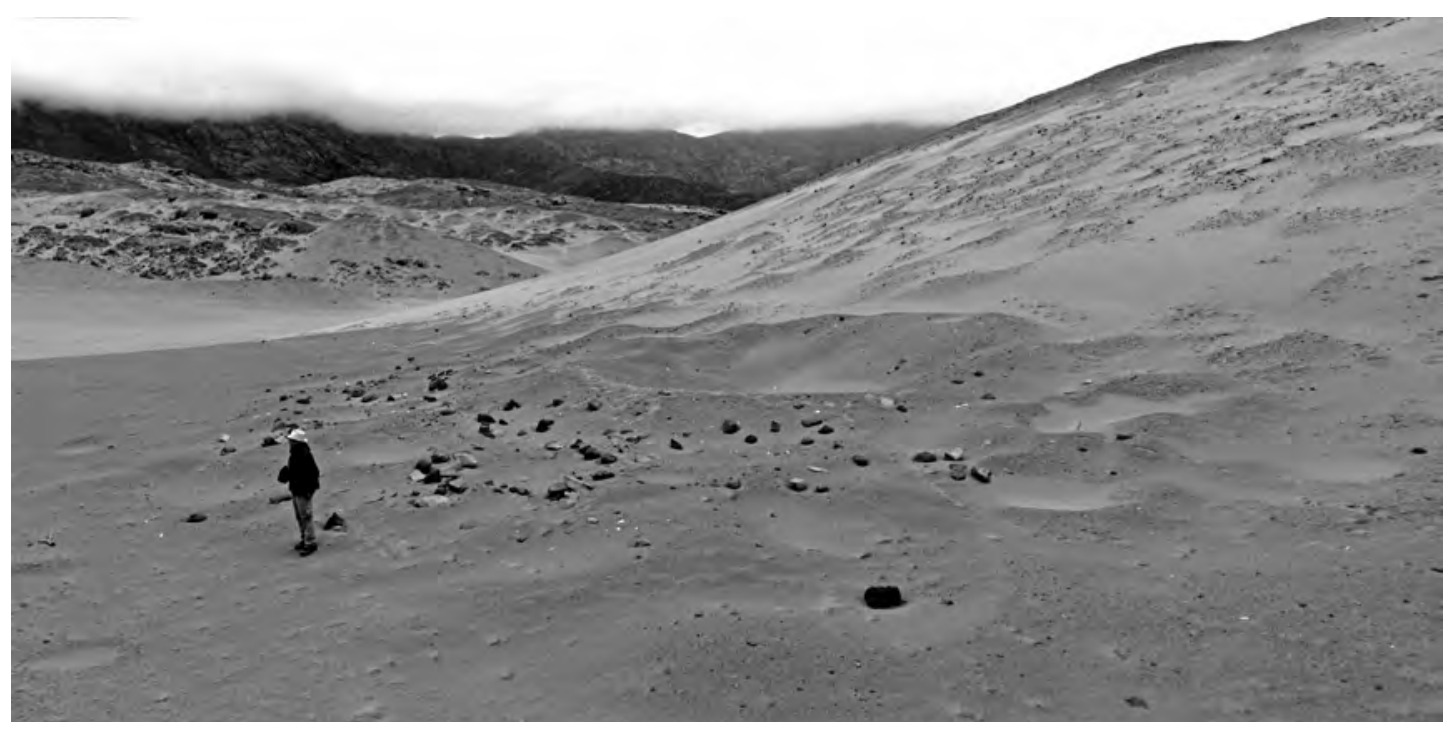

Figura 15. Vista del cementerio arqueológico. 
Gori Echevarría, Víctor Corcuera y Eduardo León / Un camino transversal al Qhapaq Ñan...

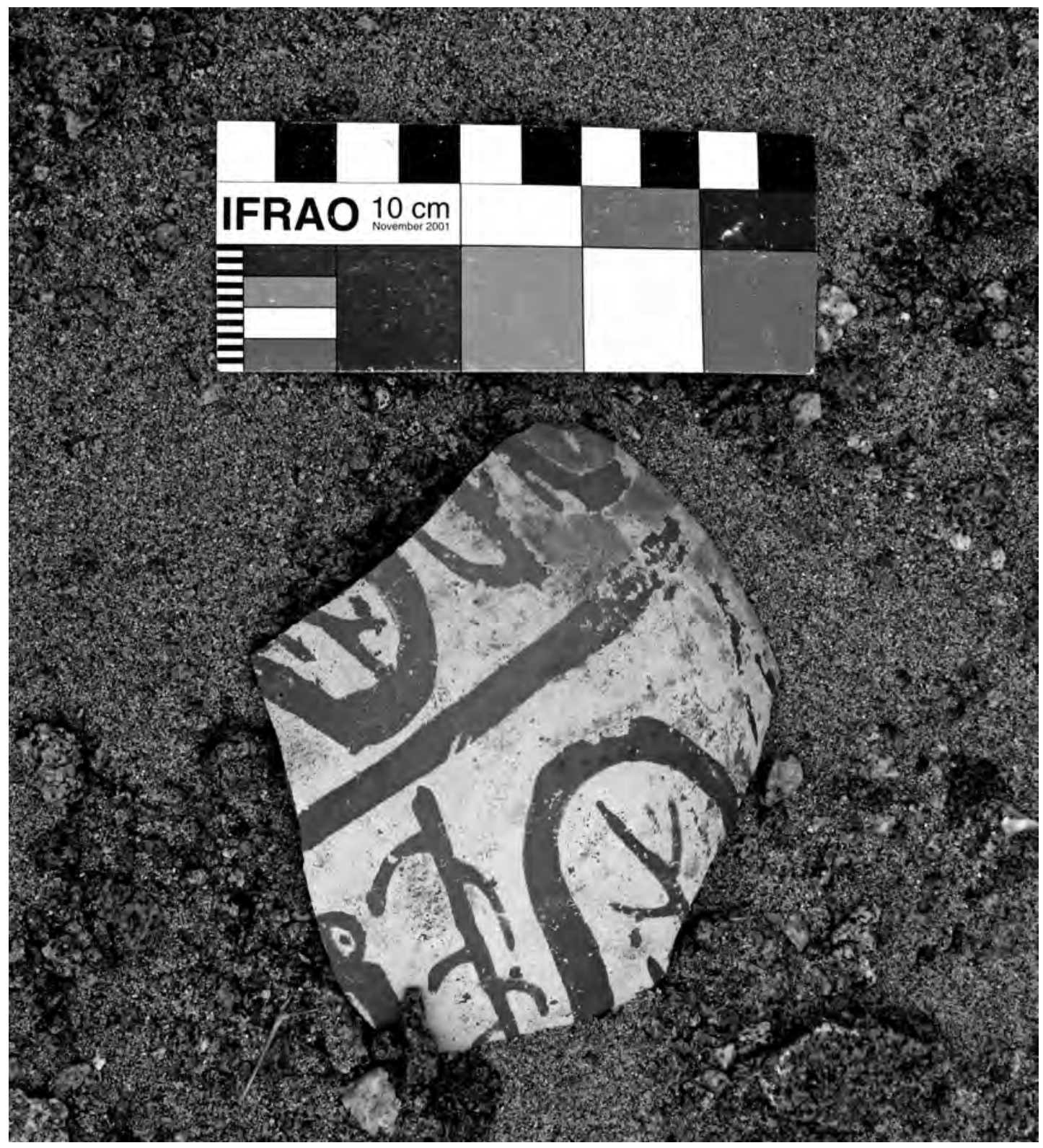

Figura 16. Fragmento de cerámica Moche, in situ. 


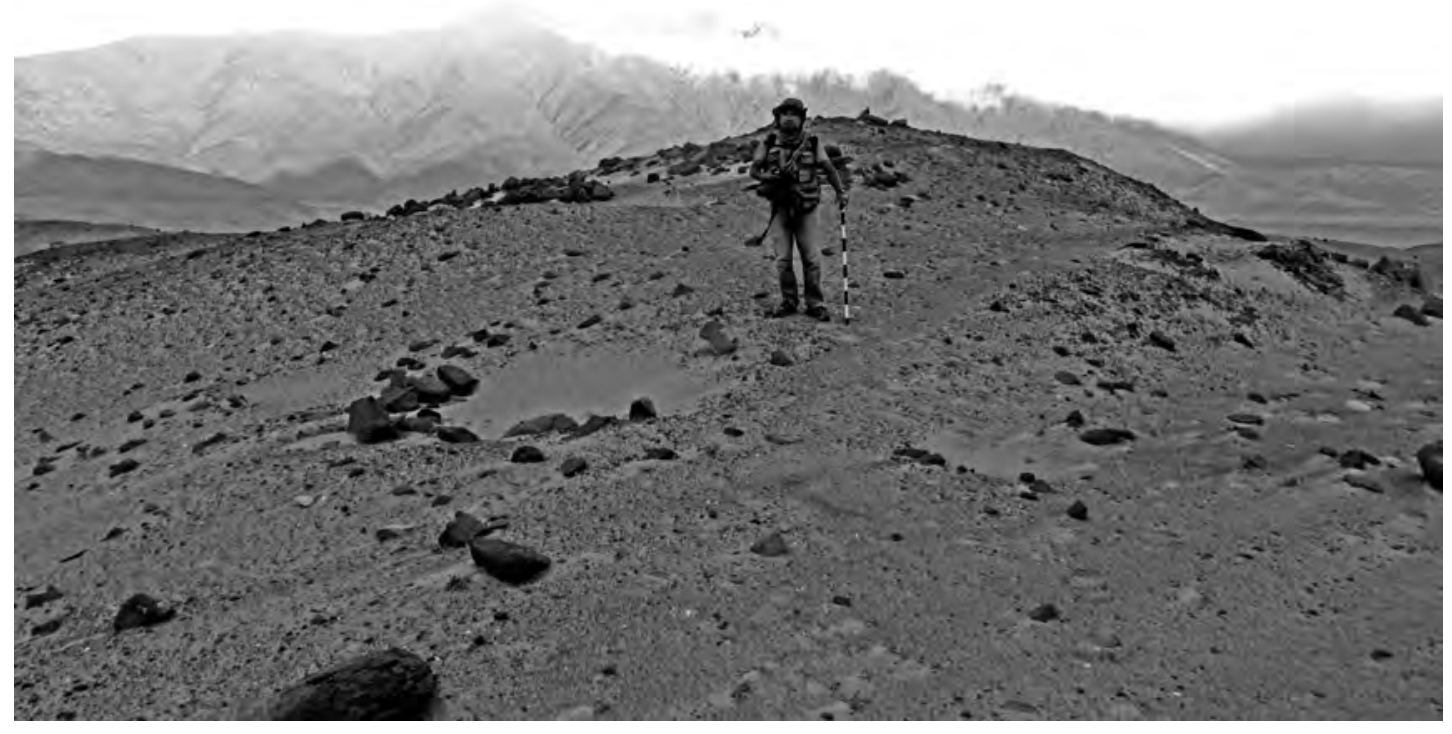

Figura 17. Edificaciones arqueológicas de piedra.

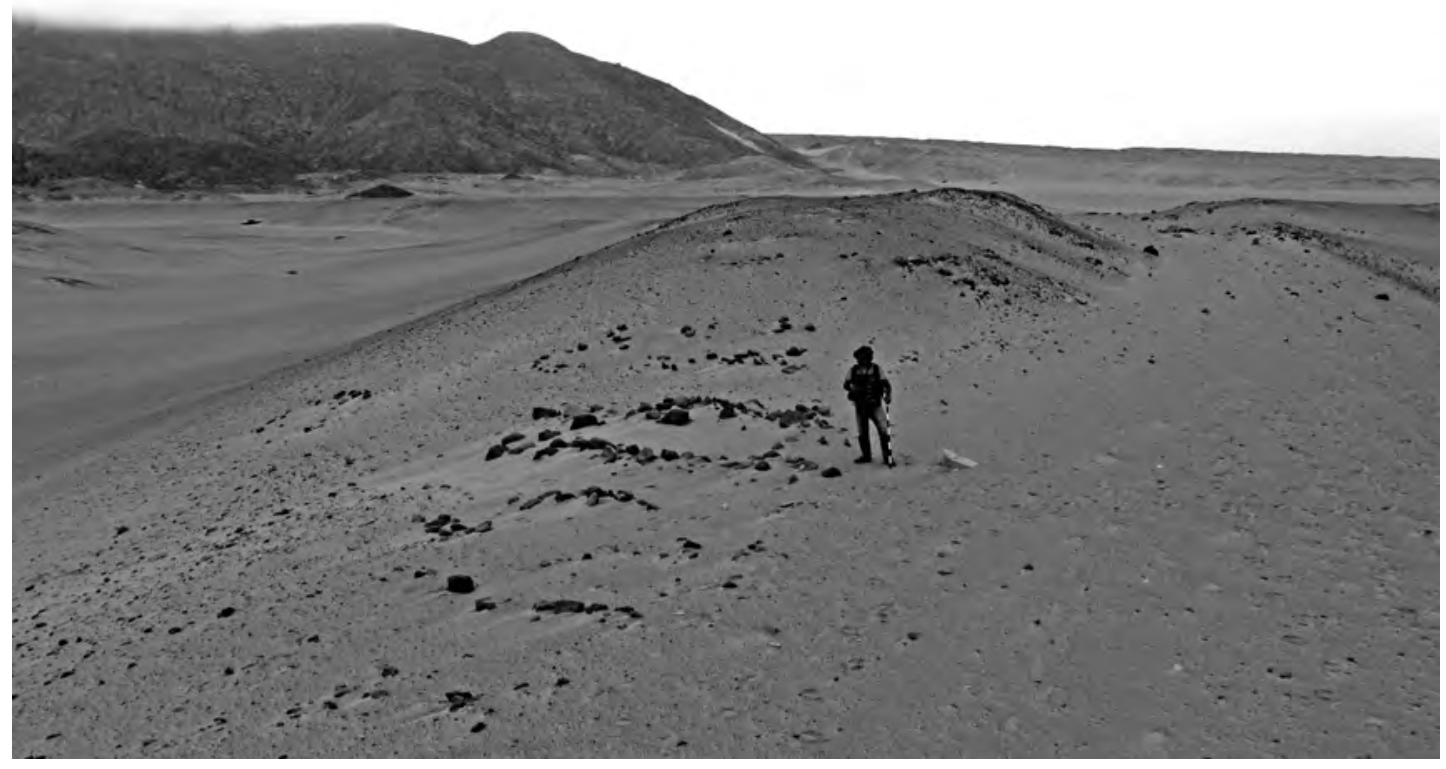

Figura 18. Edificaciones arqueológicas de piedra. 
Gori Echevarría, Víctor Corcuera y Eduardo León / Un camino transversal al Qhapaq Ñan...

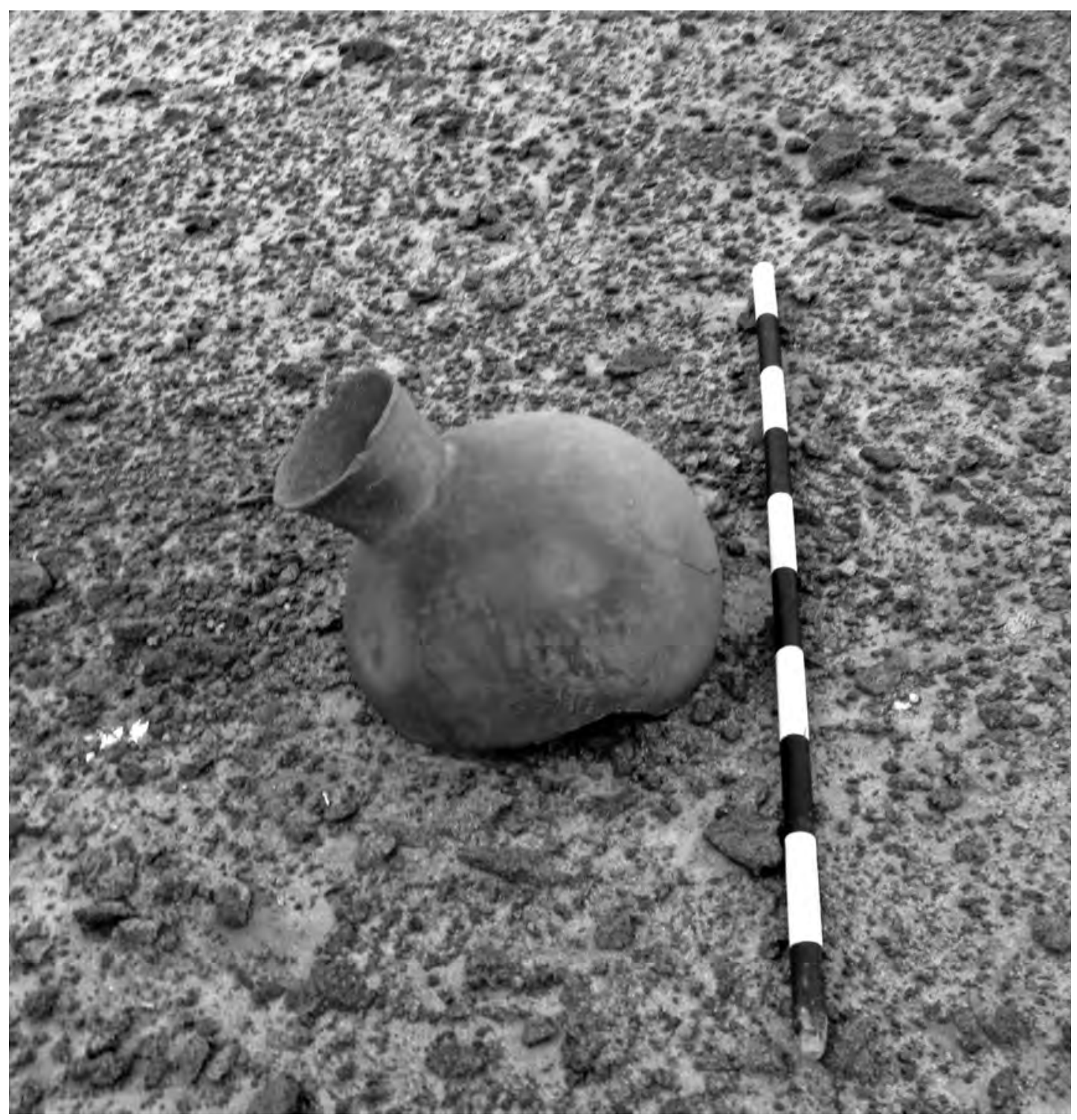

Figura 19. Cerámica tardía aislada cerca del camino. 
